\title{
A Adaptabilidade de NPCs em Jogos com Estilo Presa-Predador Usando Algoritmo Genético
}

\author{
Rafael Melo Silva \\ rafams147@gmail.com \\ FACOM - Universidade Federal de Uberlândia \\ Uberlândia, Minas Gerais
}

\author{
Christiane R. S. Brasil \\ christiane.ufu@gmail.com \\ FACOM - Universidade Federal de Uberlândia \\ Uberlândia, Minas Gerais
}

\begin{abstract}
Recently there has been a major effort to improve intelligence and adaptability in games to satisfy modern gamers. In this sense, the games area has focused on the application of artificial intelligence algorithms, especially genetic algorithms. This paper shows an approching using a genetic algorithm to improve the adaptation of non-player characters (NPCs) in a prey-predator style game, presenting good results.
\end{abstract}

\section{KEYWORDS}

Algoritmos Genéticos, Presa-Predador, jogos, Non Player Characters

\section{INTRODUÇÃO}

O desenvolvimento de jogos, durante muito tempo, teve como foco principal as melhorias nas resoluções gráficas, buscando um maior realismo nos personagens e cenários. No entanto, ao se atingir um certo nível de realismo foi percebido que os jogadores almejavam jogos que possuíssem uma jogabilidade mais bem estruturada e inteligente [1].

Neste contexto, as técnicas de Inteligência Artificial (IA) têm mostrado grande importância no aprimoramento da jogabilidade, especialmente no aperfeiçoamento da inteligência dos Non-Player Characters (NPCs) [2]. Dentro da IA, destacam-se os Algoritmos Genéticos (AGs) por serem diferentes de outras técnicas, como as Máquinas de Estado Finitas, que tem um crescimento muito grande na sua complexidade na tentativa de cobrir todos os cenários possíveis sendo então não adaptáveis a cenários inesperados [3]. Os AGs [4] imitam o processo de seleção natural que ocorre entre as espécies na natureza. No AG, existe uma população em que seus indivíduos representam possíveis soluções de um dado problema. A partir da aplicação de operadores de reprodução (cruzamento ou crossover, e mutação) [5] novas gerações da população são geradas, até que um critério de parada seja atingido (a solução ótima ser encontrada ou um número de gerações ser alcançado).

Neste trabalho foi usado um jogo com modelo presa-predador. Dentre esses tipos de jogos pode-se citar como exemplos: o jogo Calangos [6], que tem uma modelagem bem realista entre animais da fauna brasileira e o jogo ARK: Survival Evolved [7], lançado em 2017, mas com acesso antecipado desde 2015 tendo vendido mais de 5,5 milhões de cópias no primeiro ano desse período antes mesmo do lançamento oficial [8]. Este último é um jogo de sobrevivência em um mundo de mapa aberto com dinossauros e outras criaturas pre-históricas com um ecossistema de várias espécies diferentes possuindo entre elas relações presa-predador. É importante destacar que não somente jogos com a relação explícita presa-predador se encaixam no modelo presa-predador, podendo haver uma generalização para jogos com objetivo de captura e evasão, tais como o clássico Pac-man [9] ou jogos modernos como os da franquia Resident Evil [10] e o multiplayer Dead By Daylight [11], sendo este um exemplo de jogo presa-predador sem a presença de NPCs.

Nos jogos com modelagem presa-predador existe um grupo de personagens, considerados os predadores, que buscam caçar, consumir ou matar um outro grupo de personagens, que representam as presas, e que possuem como objetivo fugir dos predadores [12]. Um jogo desse tipo pode ser modelado como um sistema multi-agente, onde os predadores e presas seriam controlados por agentes inteligentes [13], sendo estes definidos como entidades que percebem o ambiente e agem sobre ele [14]. Logo a técnica de AGs pode ser aplicada para controlar a tomada de decisão desses agentes inteligentes, como os NPCs.

Inspirado no jogo de [12], este trabalho utilizou um Algoritmo Genético para controlar a adaptabilidade de NPCs em um jogo do tipo presa-predador durante sua execução.

\section{SOLUÇÃO PROPOSTA}

Foi desenvolvido um jogo com inspiração no trabalho de [12], no qual é aplicado um Algoritmo Genético para controlar a adaptabilidade de NPCs. O jogo desenvolvido neste trabalho possui 3 tipos de NPCs, referenciados aqui como NPC1, NPC2 e NPC3, sendo estes distribuídos em níveis de uma cadeia alimentar, onde os de níveis superiores se alimentam dos de níveis inferiores, caracterizando um ambiente presa-predador. No trabalho de [12] eram 8 tipos diferentes de NPCs.

Cada personagem possui um conjunto de atributos (dano, defesa, visão, velocidade, fome e vida), onde os valores iniciais dos atributos fome e vida são os mesmos para todos os tipos de NPCs, enquanto os outros atributos são inicializados com valores diferentes para cada tipo de NPC, sendo os valores iniciais dos atributos exibidos na Tabela 1.

Tabela 1: Atributos dos NPCs

\begin{tabular}{cccc}
\hline Atributos & NPC1 & NPC2 & NPC3 \\
\hline Nível da cadeia & 1 & 2 & 3 \\
ID & 1 & 2 & 3 \\
Dano & 0.4 & 0.56 & 1.75616 \\
Defesa & 0.4 & 0.48 & 0.82944 \\
Visão & 10 & 30 & 50 \\
Velocidade & 0 & 50 & 80 \\
Fome & 0 & 0 & 0 \\
Vida & 100 & 100 & 100 \\
\hline
\end{tabular}

Durante o jogo, os personagens se movimentam de acordo com sua velocidade e a cada passo de tempo é efetuada sua próxima 
ação, que considera o tamanho de sua fome e o nível do personagem na cadeia alimentar. As ações disponíveis são: atacar, fugir ou movimentar-se aleatoriamente, sendo que a ação de atacar resulta numa batalha entre os dois personagens envolvidos, onde ambos perdem vida, e as ações fugir ou movimentar possuem um custo no seu atributo fome que aumenta quando essas forem realizadas. Quando ocorre a morte de um NPC, por conta de sua vida ter zerado ou de seu atributo fome ter atingido o valor 120, existe uma probabilidade de ser executado o AG para evoluir o NPC morto e ser gerado um novo NPC que é então inserido no cenário, caso a probabilidade não seja contemplada é inserido no cenário um NPC com os mesmos atributos que o NPC morto. Deste modo, o AG, cujo os indivíduos são codificados como um vetor de 4 posições representando os valores dos atributos Dano, Defesa, Visão e Velocidade, tem como objetivo melhorar os atributos do NPC morto, gerando um novo personagem do mesmo tipo, mantendo os atributos num nível adaptado ao jogo e sem a perda das relações de presa e predador, de maneira que as presas não se tornem mais fortes que seus predadores. No AG são utilizados os atributos do melhor NPC da espécie do NPC morto na geração da população inicial, fazendo assim com que aquela espécie evolua a partir da sua força no jogo atualmente.

A função de aptidão (ou fitness) indica quão apta (forte) uma solução está no ambiente. Neste trabalho esta função é composta pela soma dos atributos do indivíduo $\left(V A R_{1}\right)$, e duas variáveis, uma para a média da soma dos atributos dos NPCs atualmente no jogo e que são predadores do tipo que está sendo evoluído $\left(V A R_{2}\right)$ e a outra para os que são presas do indivíduo que está sendo evoluído $\left(V A R_{3}\right)$. A fórmula utilizada é apresentada a seguir.

$$
\mathrm{Ap}=\frac{V A R_{2}}{V A R_{1}}-\frac{V A R_{3}}{V A R_{1}}
$$

\section{CONSIDERAÇÕES FINAIS}

Este trabalho foi desenvolvido como uma adaptação do jogo de [12] para um Trabalho de Conclusão de Curso, e vêm apresentando resultados preliminares promissores. No atual momento de desenvolvimento, tem-se no jogo 3 tipos de NPCs, com 4 NPC1, 3 NPC2 e 2 NPC3 e sem a presença do jogador. Como já fora mencionado, no trabalho em que este foi inspirado foram utilizados 8 tipos de NPCs, sendo essa uma das diferenças deste, também são diferentes os valores iniciais dos atributos e algumas etapas do AG, como o funcionamento da batalha que ocorre antes do cálculo do fitness, onde é checado para cada indivíduo se os valores dos seus atributos superam os valores da média dos atributos dos personagens atualmente vivos no jogo, e no funcionamento do crossover implementado, cuja diferença é detalhada a seguir.

Na execução do Algoritmo Genético foram usados os seguintes parâmetros: tamanho da população de 100 , número máximo de gerações de 100, modo de seleção dos pais sendo 2 pais aleatórios, probabilidade de crossover de $45 \%$, e probabilidade de mutação de $3 \%$. No presente trabalho, foram implementados a mutação uniforme e o crossover de um ponto, diferente do trabalho inspirado, no qual o crossover utilizado também realiza um ponto de corte, funcionando da seguinte forma: no momento da troca das partes podem ocorrer mudanças na posição destas, de forma que num ponto de corte no meio do indivíduo a primeira parte do Pai 1 pode se tornar a segunda parte do Filho 1, enquanto a segunda parte do Pai 2 se torna a primeira parte, assim ocorrendo não só a troca de partes entre os pais, mas também uma permutação de suas posições no filho resultante.

As Figuras 1 e 2 apresentam os resultados gerados neste trabalho com 3 tipos de NPCs, nelas são exibidos os valores das médias dos atributos de duas execuções do jogo, uma sem o uso do AG e uma com o uso do AG. Não foram exibidos os valores do desvio padrão pois no trabalho de [12] estes também não foram mostrados. Vale ressaltar que para o andamento do trabalho, seria interessante normalizar os dados a fim de explicitar os valores de desvio padrão. Por meio das figuras foi possível verificar a melhoria da média dos valores dos atributos com a presença do AG, ressaltando um aumento significativo dos valores da Visão e da Velocidade, mais especificamente, gerando NPCs indubitavelmente mais fortes durante a execução. Na Figura 1 é possível perceber maiores valores ao longo do tempo nos atributos de Dano e Defesa, usando o AG para evoluir o NPC, sempre que possível. Na Figura 2 esse aumento dos valores tornou-se mais notável ainda nos atributos de Visão e Velocidade resultando em uma população de NPCs mais adaptáveis ao jogo, e com isso, o jogo tende a ser mais interessante ao jogador pelo acréscimo de dificuldade. Para continuidade do trabalho, deseja-se experimentar outras funções de aptidão, a fim de lidar com a questão em que os NPCs podem evoluir a ponto de se tornar melhores que seus predadores, o que pode ser um vantagem por um lado tornando o ambiente menos previsível, mas uma desvantagem, pois estaria se alterando a hierarquia na relação presa-predador.

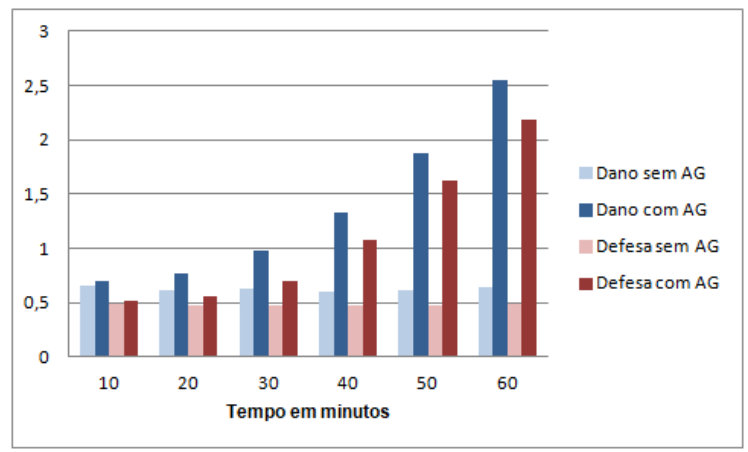

Figura 1: Dano e defesa por faixa de tempo.

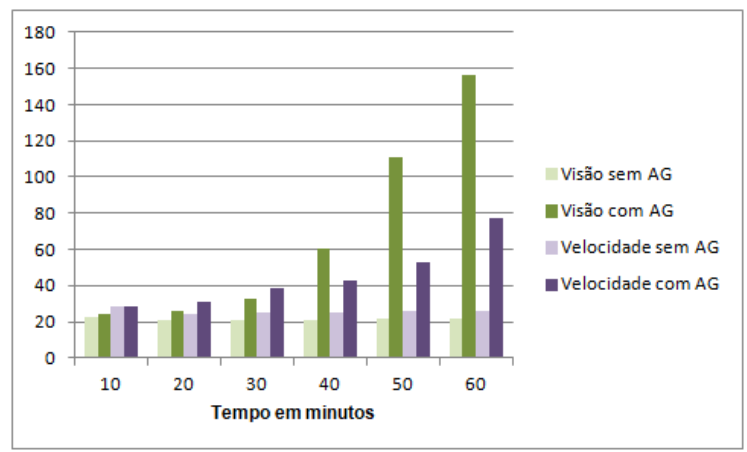

Figura 2: Visão e velocidade por faixa de tempo. 


\section{REFERÊNCIAS}

[1] Márcio Kassouf Crocomo. Um algoritmo evolutivo para aprendizado on-line em jogos eletrônicos. Master's thesis, Universidade de São Paulo, 2008.

[2] Su-Hyung Jang, Jong-Won Yoon, and Sung-Bae Cho. Optimal strategy selection of non-player character on real time strategy game using a speciated evolutionary algorithm. In 2009 IEEE Symposium on Computational Intelligence and Games, pages 75-79, 2009.

[3] Penelope Sweetser and Janet Wiles. Current ai in games: a review. Australian Journal of Intelligent Information Processing Systems, 8(1):24-42, 2002.

[4] John H. Holland. Adaptation in Natural and Artificial Systems: An Introductory Analysis with Applications to Biology, Control and Artificial Intelligence. MIT Press, 1992.

[5] Paulo Henrique Ribeiro Gabriel and Alexandre Cláudio Botazzo Delbem. Fundamentos de algoritmos evolutivos. ICMC-USP, 2008. URL http://conteudo.icmc. usp.br/CMS/Arquivos/arquivos_enviados/BIBLIOTECA_113_ND_75.pdf.

[6] Angelo C. Loula, Leandro N. De Castro, Antônio L. Apolinário, Pedro L.B. Da Rocha, Maria Da Conceição L. Carneiro, Vanessa Perpétua G.S. Reis, Ricardo F. Machado, Claudia Sepulveda, and Charbel N. El-Hani. Modeling a virtual world for the educational game Calangos. International fournal of Computer Games Technology, 2014, 2014. ISSN 16877055. doi: 10.1155/2014/382396.
[7] ARK. Ark: Survival evolved, 2015-2019. URL http://playark.com.

[8] VOXEL. Ark: Survival evolved surpreende nas vendas para pc e xbox one, August 2016. URL https://www.voxel.com.br/noticias/ark-survival-evolved-surpreendevendas-pc-xbox-one_824585.htm.

[9] Georgios N. Yannakakis. AI in Computer Games: Generating Interesting Interactive Opponents by the use of Evolutionary Computation. $\mathrm{PhD}$ thesis, University of Edinburgh. College of Science and Engineering. School of Informatics., 2005. URL https://era.ed.ac.uk/handle/1842/879.

[10] Resident Evil. Resident evil portal, 1996-2019. URL http://www.residentevil.com/.

[11] Dead by Daylight. Dead by daylight, 2015-2019. URL https://deadbydaylight. com/en.

[12] Ricardo Cherobin. Aplicação do modelo da cadeia alimentar juntamente com algoritmos genéticos para criação de npcs adaptativos. Master's thesis, Universidade do Vale do Itajaí, 2014. URL https://siaiap39.univali.br/repositorio/handle/ repositorio/1025.

[13] Eduardo D. M. Dias, Marley M. B. R. Vellasco, and André V. Abs da Cruz. Modelo Neuro-Evolutivo Com Inspiração Quântica Aplicado Ao Problema Presa Predador. Number March, pages 1-7, 2016. doi: 10.21528/cbic2011-25.6.

[14] Stuart J. Russel and Peter Norvig. Artificial Intelligence: A Modern Approach. Prentice Hall, 2003 